\title{
Zinc as a risk factor for myopia: Can we accurately assess status?
}

\section{Abstract}

Myopia is one of the leading causes of preventable blindness in the world. Its prevalence has risen drastically over recent decades, and it is estimated that close to half the world population will be myopic by 2050 . The rise in myopia is lifestyle related. Myopia occurs as a consequence of excessive eye growth, which may be related to general growth. Diet, therefore, is a potential risk factor. A number of Asian studies have reported lower levels of zinc in myopic adolescents, when compared to controls. Currently, there are no reliable indicators of zinc status. This study explores the association between zinc status and myopia using a combination of zinc assessment methods.

Participants from two different population-based studies were used. Data from 1,095 adolescents aged 12-19 years, from the US National Health and Nutrition Examination Study were used to examine the relationship between dietary zinc intake and myopia. Data from 304 subjects of similar age from the Korean National Health and Nutrition Examination Study were used to examine the association between serum zinc levels and myopia using multivariate logistic regression.

$43 \%$ (NHANES) and 84\% (KNHANES) of subjects were found to be myopic. Mean dietary intake of zinc was lower among myopes relative to non-myopes, but not significantly. In multivariate logistic regression, dietary zinc was not significantly associated with myopia. Among Korean subjects mean serum zinc was found to be higher in non-myopes v. myopes $(p=0.809)$. Multiple logistic regression did not show any significant relationship between serum zinc and myopia, after adjustment for confounders.

In contrast to previous studies, no relationship was found between lower dietary zinc intake or lower serum zinc, and myopia. Currently the BOND Zinc Expert Panel recommend plasma zinc concentration to assess zinc status, despite its extreme sensitivity to both internal and external factors like; inflammation, fasting, pregnancy, oral contraception and diurnal rhythm. Biological samples are very easily contaminated, meaning strict quality controls and procedures are required. These factors severely impact reliability, and thus, comparison between studies is challenging, particularly in the case of contrasting findings.As zinc is a vital micronutrient and an estimated one-third of the population are affected by zinc deficiency. A reliable biomarker of status is important, for clinical and research needs.

\section{Conflict of Interest}

There is no conflict of interest 